\title{
Enhancing Ex-Situ Lift-Out with EXpressLO ${ }^{\mathrm{TM}}$
}

\section{L.A. Giannuzzi}

L.A. Giannuzzi \& Associates LLC, 12580 Walden Run Dr, Fort Myers, FL 33913 USA.

Ex-situ lift out (EXLO) of focused ion beam (FIB) prepared transmission electron microscopy (TEM) specimens are a high throughput and reproducible method [1]. In addition to being faster, since EXLO is performed outside of the FIB-based instrument, the technique does not require costly instrumentation time. The innovation of EXpressLO ${ }^{\mathrm{TM}}$ negates the need for a thin carbon specimen support film and allows for additional FIB milling if necessary [2,3]. In addition, EXpressLO ${ }^{\mathrm{TM}}$ allows for easy backside manipulation which avoids curtaining artifacts [3].

FIG. 1(a) shows a $1500 \mathrm{~nm}$ thick specimen that has been FIB milled free and manipulated outside of the FIB via EXLO into a backside orientation onto a $\mathrm{Cu}$ EXpressLO ${ }^{\mathrm{TM}}$ grid. The specimen sticks directly to the $\mathrm{Cu}$ grid surface via Van der Waals forces. The slotted grid support negates the need for a carbon support film. The specimen is oriented such that the Si substrate faces the open portion of the slot which allows for subsequent FIB thinning in this backside orientation. The specimen was indeed further FIB milled to electron transparency in this backside orientation. FIG. 1(b) shows a TEM image of the final FIB milled EXpressLO ${ }^{\mathrm{TM}}$ specimen. Since the specimen was FIB milled from the Si or backside, there are no curtaining artifacts from the patterned metal layers into the $\mathrm{Si}$ substrate.

FIG. 2(a) shows ex-situ lift-out of a specimen FIB milled to electron transparency and manipulated onto an EXpressLO ${ }^{\mathrm{TM}}$ grid outside of the FIB. After manipulation to the EXpressLO ${ }^{\mathrm{TM}}$ grid, it was analyzed via TEM and imaged as per FIG. 2(b). Since in this case the specimen was FIB milled to electron transparency prior to EXLO, curtaining artifacts from the patterned metal layers are indeed observed within the $\mathrm{Si}$ substrate. The EXpressLO ${ }^{\mathrm{TM}}$ method and grid design combines the high throughput of EXLO with the ability to further FIB or ion mill after manipulation [4].

\section{References}

[1] L.A. Giannuzzi and F.A. Stevie (eds.) Introduction to Focused Ion Beams, (2005) Springer.

[2] L.A. Giannuzzi, Microsc. Microanal. 18 (Suppl 2) (2012) 632-633.

[3] Lucille A. Giannuzzi, ISTFA Conf. Proc., (2012) 388-390.

[4] This technique and grid design is patent pending. 
(a)

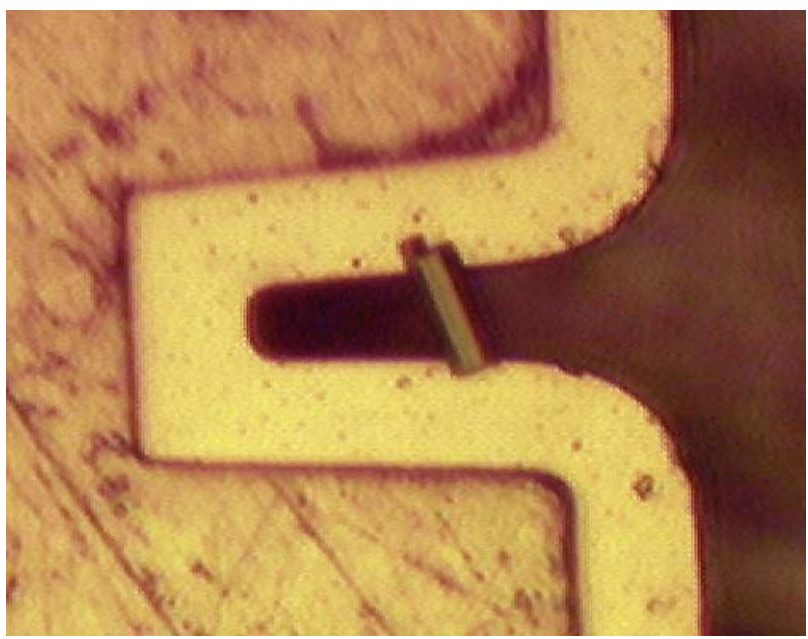

(b)

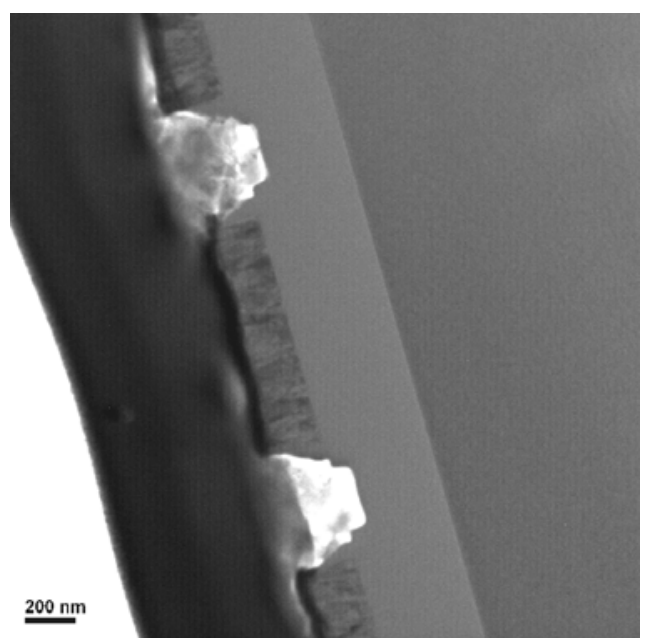

FIG. 1. (a) Ex-situ lift-out of a $1500 \mathrm{~nm}$ thick specimen manipulated into a backside orientation onto an EXpressLO ${ }^{\mathrm{TM}}$ grid. (b) TEM image of specimen after backside FIB thinning to electron transparency showing no curtaining artifacts.

(a)

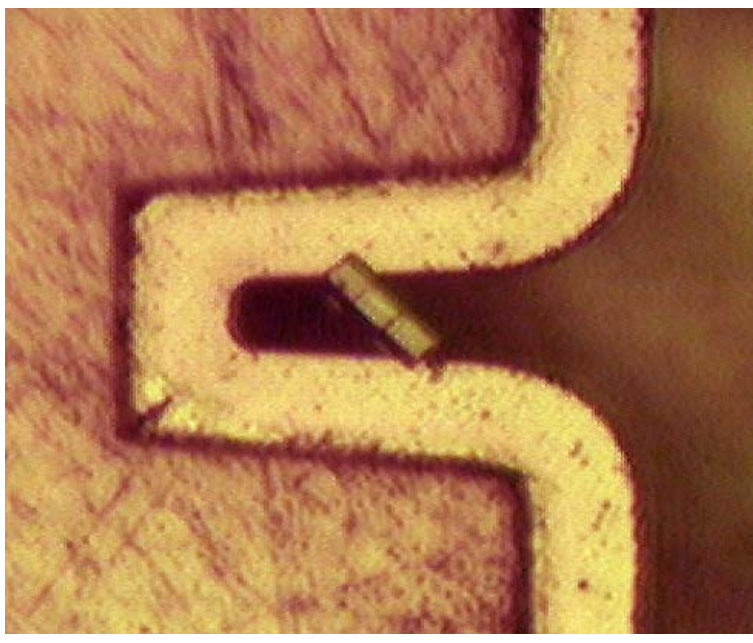

(b)

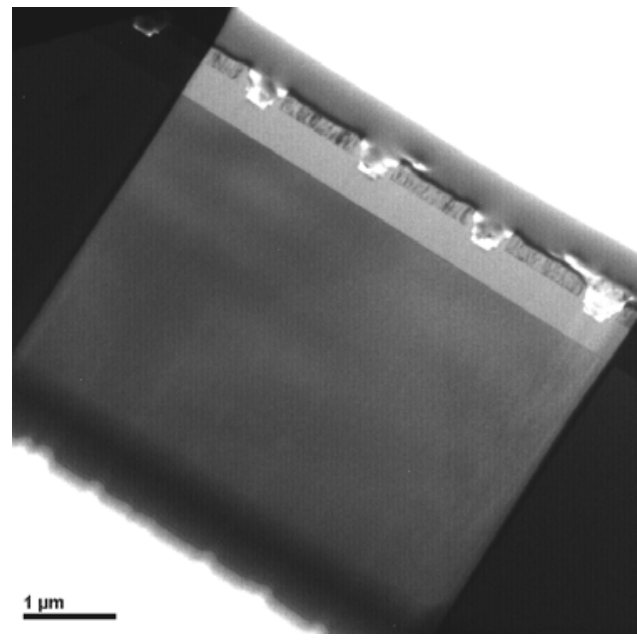

FIG. 2. (a) Ex-situ lift-out of a specimen FIB milled to electron transparency and manipulated onto an EXpressLO ${ }^{\mathrm{TM}}$ grid. (b) TEM image of the specimen in (a) directly manipulated onto an EXpressLO ${ }^{\mathrm{TM}}$ grid. 